\title{
ACTITUD DE LOS ESTUDIANTES UNIVERSITARIOS ANTE LA PLATAFORMA MOODLE
}

\section{ATTITUDE OF UNIVERSITY STUDENTS TOWARDS THE MOODLE PLATFORM}

\author{
Dra. Guadalupe A. Maldonado Berea ${ }^{1}$ \\ gumaldonado@uv.mx \\ Dra. Esther Vega Gea ${ }^{2}$ \\ esther.vega@uco.es \\ ${ }^{1}$ Universidad Veracruzana. Dirección General de la Unidad de Estudios de Posgrado. \\ Diego Leño $N^{o}$ 43, 91000, Zona Centro, Xalapa, Veracruz (México) \\ ${ }^{2}$ C/ Periodista Quesada Chacón $N^{o} 135^{\circ}, 14005$, Córdoba (España)
}

El desarrollo de sistemas de Teleformación en la enseñanza universitaria ha puesto de manifiesto cómo el diseño de nuevas estrategias educativas vendrá determinado por aspectos como la actitud que los receptores de la acción docente tienen ante ellos. En este artículo presentamos los resultados obtenidos a través de un estudio de tipo correlacional desarrollado en la Facultad de Ciencias de la Educación de la Universidad de Córdoba, sobre la actitud de los estudiantes ante la plataforma Moodle. El principal resultado alcanzado es que el alumnado, en general, tiene una actitud positiva hacia el uso de la plataforma de Teleformación Moodle.

Palabras clave: Moodle, alumno universitario, enseñanza superior, formación.

The development of e-learning systems in university education has shown that the design of new strategies will be determined by factors such as attitudes between recipients of the teaching action. In this paper we present the results obtained through a correlational study developed at the Faculty of Educational Sciences, University of Córdoba, on the attitude of students towards the Moodle platform. The main result obtained is that students generally have a positive attitude toward using Moodle E-learning platform. Keywords: Moodle, university pupils, higher school, training. 


\section{Introducción.}

Los nuevos entornos formativos emplearán cada vez más y en mayor medida las TIC, lo que hace que tanto profesores como estudiantes observen un mínimo de competencias tecnológicas que les permitan desenvolverse de una mejor manera en estos entornos virtuales. Esto genera un nuevo tipo de alfabetización, que «se centre no sólo en los medios impresos y sus códigos verbales, sino también en la diversidad de medios con que nos encontramos y en los códigos que los caracterizan» (Gisbert, Cabero \& Llorente, 2007, p.266). La alfabetización del siglo XXI muestra que los nuevos enfoques subrayan las competencias para utilizar la información y el conocimiento, mostrando de esta forma su carácter multidimensional.«Profesores, estudiantes, trabajadores y ciudadanos deben incorporar desde ahora los siguientes elementos para desarrollar sus conocimientos y capacidad de razonamiento crítico» (Varis, 2005, p.2):

- Alfabetización tecnológica: la capacidad de utilizar nuevos medios, como Internet, para acceder a la información y comunicarla eficazmente.

- Alfabetización informacional: la capacidad de recopilar, organizar y evaluar la información y formarse opiniones válidas basadas en los resultados.

- Creatividad mediática: la capacidad, cada vez mayor, de los ciudadanos de producir y distribuir, donde quiera que se encuentren, contenidos para audiencias de todos los tamaños.

Alfabetización global: consiste en comprender la interdependencia entre las personas y los países y tener la capacidad de interactuar y colaborar eficazmente a través de las culturas.
Alfabetización responsable: la competencia de examinar las consecuencias sociales de los medios de comunicación desde el punto de vista de la seguridad, la privacidad y otros.

Por tanto, este alfabetismo en medios y TIC construye una visión de la importancia que tienen los medios en la sociedad actual así como en la generación de habilidades que posibiliten la indagación y la auto expresión que es tan necesaria para los ciudadanos de una sociedad democrática, por lo que esta alfabetización deberá capacitar al ciudadano en una serie de aspectos y competencias.

En este caso, el rol del estudiante hoy día con respecto al uso de las TIC (Marqués, 1999, 2011; Vázquez, 2011), sea dentro de lo más representativos:

- Usar las TIC para procesar la información y como instrumento cognitivo.

- Usar las TIC para comunicarse en el ciberespacio, ampliando su entorno de relación.

- Usar las fuentes digitales de información para el aprendizaje, desarrollando estrategias para buscar, valorar, guardar, estructurar y aplicar la información.

- Aprender en la red, con los entornos virtuales.

- Trabajar de forma individual y colaborativa.

- Responsabilizarse del aprendizaje y auto dirigirlo.

- Estar motivado, trabajar de manera continuada con intensidad y perseverancia.

- Aceptar la incertidumbre y la ambigüedad.

- Pensar críticamente y actuar con reflexión.

- Ser creativo y estar abierto al cambio.

Las Plataformas virtuales se emplean como sinónimo de la formación a través de la red o de redes telemáticas así como también la Teleformación, e-learning, web-based 
learning, entre otras. Estas son consideradas como una modalidad de formación dentro de la educación a distancia (Llorente \& Cabero, 2009).

Con la aparición de TIC, como la televisión digital, la radio digital o el video y más recientemente la web 2.0, la EA (educación abierta) se redefine y da paso a la ED (educación a distancia), que al incorporar de forma intensiva las TIC en la educación, principalmente por el uso de la simulación, la realidad virtual y la multimedia, se transforma la ED en Educación Virtual (EV), sumándose al «no tiempo»y «no espacio». Y dentro de esta, surgen los programas de aprendizaje o cursos en línea, que son desarrollados en diversas plataformas, cuya característica distintiva es el apoyo en Internet como medio de enlace entre el profesor y el estudiante (Herrera, 2002).
Siendo en esta última donde se ubica la Teleformación (sinónimo de e-learning para este trabajo) que apoyado por el uso de las Plataforma Virtuales, "proporciona la oportunidad de crear ambientes de aprendizaje centrados en el estudiante» (Boneu, 2007, p. 37) que es uno de los ejes principales del EEES, y que es el contexto en el que se ha ubicado este estudio.

\section{Metodología.}

Como objeto de estudio, se ha tomado a la plataforma de Teleformación Moodle, ya que es la que se emplea en la UCO, es un LMS (Learning Management System), que se distribuye gratuitamente como Software libre. La palabra Moodle es un acrónimo de Modular Object-Oriented Dynamic Learning Environment(Entorno de Aprendizaje Dinámico Orientado a Objetos y

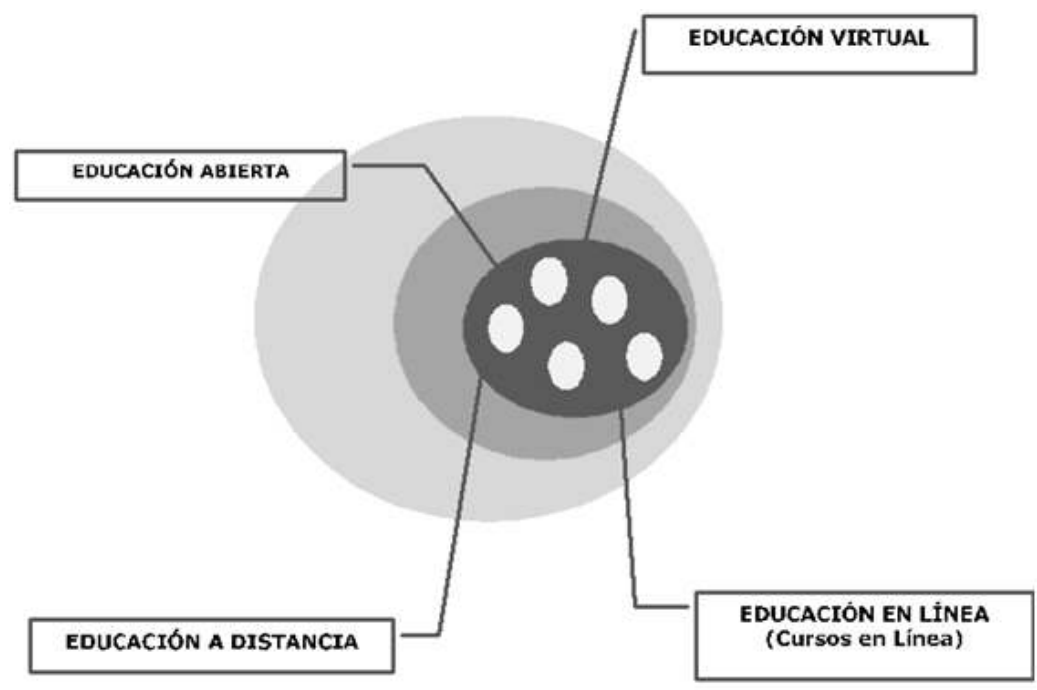

Figura 1.Delimitación de la Educación abierta, a distancia y virtual (Herrera, 2002) 


\begin{tabular}{|c|c|c|}
\hline \multirow[b]{2}{*}{$\begin{array}{l}\text { ESTRATEGIAS } \\
\text { DIDÁCTICAS }\end{array}$} & APOYO AL APRENDIZAJE & \multirow{2}{*}{$\begin{array}{c}\text { SOPORTE } \\
\text { Herramientas del } \\
\text { curso } \\
\end{array}$} \\
\hline & $\begin{array}{cc}\text { Herramientas de } & \text { Herramientas del } \\
\text { Comunicación } & \text { Estudiante } \\
\end{array}$ & \\
\hline \multicolumn{3}{|l|}{ Método expositivo } \\
\hline $\begin{array}{l}\text { Dinámica de } \\
\text { grupos }\end{array}$ & & \\
\hline Simulaciones & & \\
\hline Individualización & & \\
\hline
\end{tabular}

Tabla 1. Estrategias didácticas (Marín \& Maldonado, 2010)

Modular). Creada por Dougiamas (2002), quien continúa dirigiendo el proyecto, bajo la filosofía de dar soporte a un marco de educación social constructivista. En este caso, Moodle proporciona las siguientes posibilidades técnicas para poder llevar a cabo el aprendizaje:

- Apoyo al aprendizaje, son aquellas relacionadas con la comunicación (sincrónica y asincrónica), la productividad (calendario, orientación y búsqueda) y las del estudiante (trabajo en grupo, autoevaluación, herramientas web 2.0).

- Soporte, se distinguen por estar dirigidas a la administración (autenticación, autorización de cursos, servicios host e integración del registro), al curso (herramientas de evaluación, gestión del curso, soporte técnico, gestión de alumnos, y al plan de estudios (invitados, guías docentes, diseño personalizado, editor de materiales, estándares).

En cuanto a las posibilidades didácticas que ofrece, se han establecido en base a la clasificación de las estrategias didácticas (Álvarez, 2009), aunadas a la categorización de las herramientas anteriormente mencionadas (ver Tabla 1).

Una vez revisada la literatura, se planteó como problema objeto general de estudio: comprobar las actitudes del estudiante de la Facultad de Ciencias de la Educación de la
Universidad de Córdoba de primer año sobre el uso que hace de la plataforma de Teleformación «Moodle» y su relación con las competencias tecnológicas que le son requeridas en la actualidad para su desempeño profesional. A partir de este, se establecen los objetivos específicos siguientes así como las hipótesis y las variables de partida:

Objetivos:

1. Diagnosticar las habilidades tecnológicas que posee el alumnado de primer año de la Facultad de Ciencias de la Educación en el uso de la plataforma de Teleformación Moodle.

2. Determinar las actitudes que poseen los estudiantes de la Facultad de Ciencias de la Educación de nuevo ingreso con respecto a los procesos técnicos de la plataforma de Teleformación Moodle.

3. Mostrar las actitudes que tiene el alumnado de primer año de la Facultad de Ciencias de la Educación con relación a los procesos didácticos de la plataforma de Teleformación Moodle.

Hipótesis:

1. Existe relación positiva significativa entre la actitud que tiene el alumnado de primer año de la Facultad de Ciencias de la Educación de la UCO hacia la plataforma de Teleformación Moodle y las habilidades tecnológicas que poseen. 


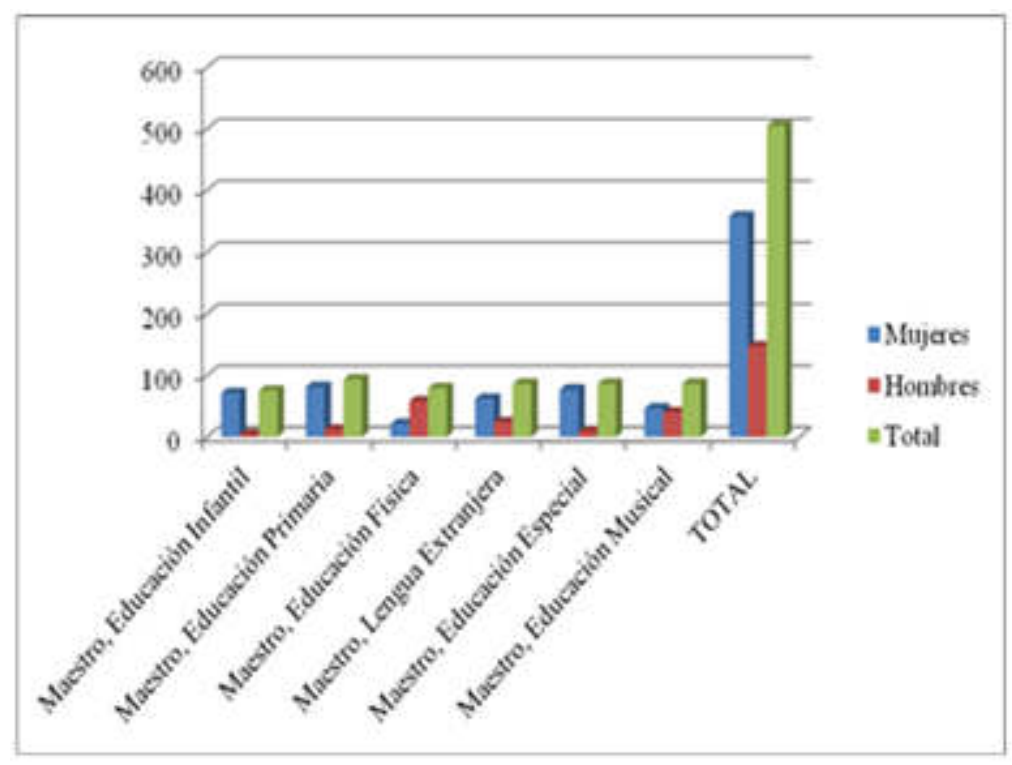

Grafica 1. Distribución de la muestra

2. Existe relación positiva significativa entre la actitud que tiene el alumnado de primer año de la Facultad de Ciencias de la Educación de la UCO y la plataforma de Teleformación Moodle con respecto a los procesos técnicos de la misma.

3. Existe relación positiva significativa entre la actitud que tiene el alumnado de primer año de la Facultad de Ciencias de la Educación de la UCO y la plataforma de Teleformación Moodle con respecto a los procesos didácticos que esta posee.

4. Existe relación positiva significativa entre la actitud que tiene el alumnado de primer año de la Facultad de Ciencias de la Educación de la UCO y la plataforma de Teleformación Moodle con respecto a ser un apoyo en el proceso de enseñanzaaprendizaje.

\subsection{Diseño de la investigación.}

Se unificaron los enfoques cuantitativo y cualitativo, a través de una metodología mixta, incorporando las aportaciones que proporciona el paradigma positivista, a través de un diseño no experimental tipo correlacional y con relación al paradigma cualitativo, un diseño de tipo fenomenológico.

\subsection{Población y Muestra.}

La población participante en este estudio fueron los estudiantes matriculados en primer año durante el período escolar 2009-2010 de la Facultad de Ciencias de la Educación, período de Octubre a Diciembre de 2009. Para su selección y así conformar una muestra homogénea se realizó un muestreo aleatorio 
estratificado, empleando como criterio de selección la presencia de los alumnos en el aula el día en que se administró el instrumento, junto con la franja horaria proporcionada por los docentes para aplicar los cuestionarios en ese momento, conformándose finalmente por 287 sujetos, pertenecientes a las titulaciones de: Educación Infantil, Educación Primaria, Educación Física, Lengua Extranjera, Educación Especial, Educación Musical (ver Gráfica 1).

\subsection{Instrumentos.}

Para la recogida de información se elaboraron dos tipos de instrumentos, un cuestionario para los estudiantes del primer año del ciclo académico 2009 - 2010 y la realización de un grupo de discusión, uno dirigido al profesorado y otra a los estudiantes de primer año. Con respecto al cuestionario aplicado a los estudiantes, se empleó la Técnica de la encuesta estructurada y el Cuestionario como instrumento. Se realizó un cuestionario preliminar que se sometió a un análisis de fiabilidad y validez, en base a los resultados obtenidos se procedió a su rediseño, generándose un cuestionario final constituido por 72 ítems integrados en 6 dimensiones:

1. Actitudes generales hacia la Teleformación.

2. Experiencia previa en Teleformación.

3. Conocimientos de herramientas de interacción.

4. Habilidades en el uso de las herramientas de interacción.

5. Procesos técnicos de la PTM.

6. Procesos pedagógicos derivados de la PTM.

Una vez aplicado el instrumento e integrada la base de datos, se procedió a realizar un análisis de fiabilidad y validez. El modelo de fiabilidad empleado para este caso fue el Alfa de Cronbach, a través del SPSS 18.0 para Windows. Se obtuvo un valor alfa de .951 el cual Mateo (2012) considera muy alto, y que indicó un nivel elevado de estabilidad en las respuestas. Por su parte, el comportamiento de cada uno de los ítems del cuestionario (ítems escalares) revela unos coeficientes alfa en todos ellos por encima .840 lo que confirma la afirmación anterior, el instrumento en este apartado gozaba de fiabilidad.

Asimismo, se realizó el análisis de la capacidad de discriminación de los elementos del instrumento para reforzar el carácter unidimensional de la prueba (García, Gil \& Rodríguez, 1995). Con base a esto, se realizó la prueba de $t$ para muestras independientes permitiendo establecer la existencia o no de diferencia estadística (n.s. $=0.05$ ) entre los grupos que puntúan bajo y alto en los ítems de cada una de las dimensiones que integraron este cuestionario preliminar. Los valores que observan puntuaciones de «P» menores que 0.05 representan un alto poder de discriminación por parte del ítem. Sin embargo, aquellos valores de «P» iguales o mayores a 0.05 no permitían una discriminación por parte del ítem. Los resultados que se obtuvieron con esta prueba a partir de los 95 ítems iniciales y de las 6 dimensiones que lo conformaban han sido los siguientes, a partir de los resultados alcanzados el cuestionario quedando finalmente compuesto por 72 ítems, como ya hemos mencionado.

\subsection{Estrategias de análisis de datos.}

El análisis de los datos recogidos tras la aplicación del cuestionario es de carácter 
eminentemente cuantitativo siendo, en primer lugar, de carácter descriptivo básico de las variables (medidas de tendencia central y medidas de dispersión). Seguidamente, se ha procedido a realizar estudios inferenciales atendiendo a criterios de clasificación como el sexo, el curso, la procedencia, el tipo de vivienda, el dispositivo de acceso a Internet, la tipología de conexión y la frecuencia semanal y diaria de conectividad, los mismos se han realizado con el programa estadístico SPSS (v.20). Para constatar los resultados obtenidos en la aplicación de los Análisis de Varianza (ANOVA) se realizaron los procedimientos post hoc como las pruebas de Scheffé y Bonferroni, ya que como indica Morales (2011) se debe implementar el análisis de Scheffé cuando no hay hipótesis previas o existen diferencias entre el tamaño de los grupos de la muestra. En nuestro estudio es la segunda premisa la que se cumple. Este mismo autor señala, que la prueba de Bonferroni se basa en la modificación del nivel de significación en función del número de comparaciones a realizar (Morales, 2011).

\section{Resultados.}

\subsection{Actitudes ante el uso de las Plataforma de Teleformación.}

Los resultados con respecto a las actitudes van en una escala entre la indiferencia y el estar parcialmente de acuerdo con respecto al uso de las plataformas de Teleformación, en este caso, hemos identificado que dentro de esta categoría se encuentra que los estudiantes consideran internet como un recurso valioso para la docencia $(\bar{x}=3.93)$, consideran que es bueno utilizar los avances tecnológicos en la educación $(\bar{x}=3.82)$ así como también opinan que el uso de las plataformas de Teleformación son un derecho de los estudiantes $(\bar{x}=3.78)$. Otro aspecto interesante fue la actitud hacia la formación en el uso de las mismas $(\bar{x}=3.63)$ y consideran que las plataformas de Teleformación pueden ayudar a cambiar el futuro de la educación superior $(\bar{x}=3.51)$. Agrupando las actitudes de los estudiantes en función de la especialidad de estudio con respecto al uso de las plataformas de Teleformación, podemos advertir los siguientes resultados tras la realización de un Análisis de Varianza para un Factor (n.s. $=0.05$ ):

- Con respecto a la actitud que poseen los estudiantes que usan las plataformas y las seguirán usando, las diferencias significativas se encuentran en Lengua Extranjera y Educación Física sí como entre Lengua Extranjera y Educación Musical ( $\mathrm{F}=3.815, \mathrm{p}=0.003)$, ubicándose dentro de la categoría de las Plataformas de Teleformación son herramientas de apoyo significativas para la educación superior.

- Existen diferencias significativas entre Educación Musical y Educación Infantil ( $\mathrm{F}=$ 2.508, $\mathrm{p}=0.033$ ), con relación a la actitud de que los estudiantes usarán las PT si el departamento y el centro se implican, esta actitud se ubica en la categoría de las PT son herramientas que deben ser evaluadas por parte del departamento y el centro antes de ser empleadas, así mismo existe otra actitud que se resalta dentro de este grupo y está relacionada con que los estudiantes prefieren estudiar hasta donde les puede llevar el uso de las PT, sin embargo, no podemos establecer con claridad en qué grupos hay diferencias significativas.

Las plataformas de Teleformación hacen difícil separar el tiempo libre del trabajo docente, sobre todo entre Educación Infantil 
y Educación Musical ( $\mathrm{F}=3.581$, $\mathrm{p}=0.004)$, esta actitud se encuentra en la categoría de que las PT permiten un trabajo académico dinámico y flexible. Asimismo, dentro de esta categoría otra actitud en donde se observan diferencias significativas entre Educación Física y Educación Infantil así como entre Educación Infantil y Educación Musical ( $\mathrm{F}=$ 2.667, $\mathrm{p}=0.024$ ) con respecto a la actitud que tienen los estudiantes ante las PT, que las ven como una excusa estupenda para reciclarse profesionalmente. Y por último, también hay diferencias significativas entre Educación Infantil y Lengua Extranjera ( $\mathrm{F}=$ 3.194, $\mathrm{p}=0.009$ ) con respecto a que ven a las PT como herramientas que permiten hacer más relajada la clase.

Por último y para advertir si existe una estructura interna y así agrupar las actitudes en un grupo reducido de factores que sean representativos de los primeros, aplicamos la técnica del Análisis Factorial. Esto nos proporciona poder dar una mejor explicación del fenómeno que estamos estudiando y podremos de esta forma establecer un modelo explicativo de la relación que existe entre las variables objeto de estudio. El análisis comienza, una vez establecidos los métodos de extracción (componentes principales) y de rotación (varimax), seleccionando las variables que hacen referencia al concepto genérico que estamos estudiando: las actitudes ante el uso de las plataformas de Teleformación. De este modo, se incorporan al modelo un total de 28 elementos representativos del concepto anteriormente mencionado. La realización de esta prueba ha aportado un total de 4 factores que explican un total de la varianza de un $68.09 \%$, lo que presupone un buen modelo factorial y el establecimiento de unas condiciones adecuadas para su análisis e interpretación.
Igualmente, se ha llevado a cabo un estudio de fiabilidad por medio de la técnica Alfa de Cronbach para cada uno de los factores, observándose un alto grado de consistencia, carácter esencial para poder expresar que todos ellos miden con fiabilidad el concepto que se trata de explicar.

En definitiva, los factores conseguidos que tratan de aportar una explicación de las actitudes de los estudiantes ante el uso de las plataformas de Teleformación se definen del siguiente modo:

Factor 1: Herramientas de apoyo significativas para la educación superior. Las plataformas de Teleformación serán una herramienta muy empleada por las instituciones de educación superior para poder ser accesible la formación formal e informal, de cara al EEES, ya que se les considera como un recurso valioso para las diversas modalidades (presenciales, semipresenciales o en red), debido a que sus herramientas de interacción favorecen la comunicación y la interrelación entre los actores que participan en los diferentes entornos de aprendizaje, convirtiéndose de esta forma en un derecho y en una necesidad para los estudiantes y los profesionales en este siglo XXI.

Factor 2: Herramientas necesarias para el aprendizaje colaborativo. La Teleformación «proporciona la oportunidad de crear ambientes de aprendizaje centrados en el estudiante» (Boneu, 2007, p.37). Debido a que emplean como mediador al ordenador para lograr un aprendizaje flexible, apoyado en un sistema de tutorías o-line, y en las capacidades y actitudes tecnológicas de los estudiantes, permite realizar comunicaciones sincrónicas y asincrónicas que favorecen el trabajo individual como grupo, así como también es posible el uso de recursos 
multimedia, hipermedia, acceso a la información mediante el uso de navegadores web, entre otros. Con base en esto, los modelos educativos en el futuro contemplarán en su diseño actividades de aprendizaje donde la interacción, el trabajo colaborativo y el autoaprendizaje serán elementos de primera magnitud (Landeta, 2007).

Factor 3: Herramientas evaluadas por parte del departamento y el centro antes de ser empleadas. Este tercer factor considera uno de los objetivos planteados en la Declaración de Bolonia, la promoción de la cooperación europea para asegurar un nivel de calidad en el desarrollo de criterios y metodología comparables. Por la definición de las prácticas de Teleformación que se desarrollen en cualquier contexto educativo girará alrededor de un asunto clave: calidad y estandarización en el uso de las TIC, lo cual impacta en los criterios que tendrán que ser evaluados para la selección pertinente de la plataforma de Teleformación (Landeta, 2007).

Factor 4: Herramientas que facilitan el trabajo académico de forma dinámica y flexible. Las TIC incrementan en la educación las oportunidades de acceso al aprendizaje continuo y posibilitan la flexibilidad e interactividad. Los campus on-line se convierten en una herramienta fundamental para establecer la cooperación entre las diferentes instituciones educativas, desarrollo de planes de estudio conjuntos, acuerdos en evaluación, convalidación y reconocimiento de competencias así como la movilidad virtual, por lo que, las plataformas de Teleformación adquieren un carácter principal dentro del EEES (Landeta, 2007).

\subsection{Formación en el uso de plataformas.}

También valoramos la satisfacción que han tenido los estudiantes con respecto a la formación en el uso de las plataformas de Teleformación y cuáles son las competencias en términos de conocimiento e importancia que han logrado obtener a través de estos estudios.

\section{Valoración (satisfacción) de la} formación.

En relación a la valoración que hemos identificado con respecto a la formación en el uso de las plataformas de Teleformación observamos que es muy baja. Aquí se resalta en un mayor grado los cursos que se ofrecen dentro del mismo centro $(\bar{x}=2.04)$, seguidas por los cursos que se dan en la Universidad de Córdoba $(\bar{x}=1.99)$.

2. Competencias formativas respecto de plataformas.

Con respecto a las competencias que han desarrollado en ellos, en términos de conocimiento e importancia, mostramos a continuación los resultados obtenidos en cada categoría.

Con relación al conocimiento.

Aquí hemos identificado que tienen un conocimiento bajo con respecto a las competencias formativas respecto de plataformas. En este margen, la más representativa es la participación en foros/ chats $(\bar{x}=2.42)$, seguida de la de entrega de trabajos/actividades $(\bar{x}=2.35)$.

Con relación a la importancia.

La importancia que le otorgan a la formación en el uso de las plataformas de Teleformación se encuentra dentro de un intervalo de importancia baja a una importancia media, en este caso, las más significativas son las relacionadas con la entrega de trabajos/actividades $(\bar{x}=2.98)$, 
seguida por la participación en foros/chats $(\bar{x}=2.82)$.

Podemos concluir, con relación a este apartado, que el conocimiento e importancia que tienen las competencias formativas respecto de las plataformas de Teleformación por parte de los estudiantes están enfocadas en este orden respectivamente en la participación en foros/chats $(\bar{x}=2.42)(\bar{x}=$ $2.82)$, seguida por la entrega de trabajos/ actividades $(\bar{x}=2.35)(\bar{x}=2.98)$, lo que nos muestra que a pesar del bajo conocimiento y de la baja importancia que tienen para ellos las competencias formativas respecto de las plataformas de Teleformación, las herramientas de interacción en las que tienen mayor conocimiento y que le dan una mayor importancia son la participación en foros/ chats y las relacionadas con la entrega de sus trabajos/actividades.

En primer lugar, podemos iniciar esta exposición de resultados aludiendo al apartado de las posibilidades técnicas de la plataforma de Teleformación Moodle, disponibles para apoyar el proceso de aprendizaje del alumnado del centro. Aludimos a la gran relevancia que este grupo muestral otorga a las herramientas de comunicación como apoyo a su proceso de aprendizaje, destacando el correo electrónico, los foros y los chats. Sin embargo, con respecto a las herramientas de productividad realizan un escaso uso, siendo la más destacable el acceso al calendario de actividades del curso, del mismo modo están las herramientas del estudiante, en donde a pesar de la poca utilización que realizan de ellas, las que más manejan son los blogs, las páginas web personales, el espacio destinado para el trabajo colaborativo y los wikis. Con respecto a las herramientas de apoyo al proceso de aprendizaje, los estudiantes emplean de forma escasa dentro de las herramientas del curso, lo relacionado a la gestión de contenidos, la gestión de alumnos y los instrumentos de evaluación y autoevaluación, asimismo entre las herramientas de diseño del plan de estudio se encuentran las guías docentes.

En segundo lugar, hay que resaltar que las posibilidades didácticas que la plataformas de Teleformación Moodle les ofrece a los estudiantes, son escasamente empleadas por ellos. En términos de las estrategias específicas que se manejan para realizar actividades o tareas, las herramientas de comunicación son las que apoyan al método expositivo en esta situación, destacándose las destinadas a la lectura y comentario de textos. En la dinámica de grupos, las herramientas del estudiante sirven de apoyo a esta estrategia para las técnicas relacionadas con los talleres, aprendizaje basado en problemas y proyectos de aprendizaje colaborativos. Con relación a las simulaciones, las presentaciones multimedia se hacen asequibles a través de las herramientas del estudiante. Y en cuanto a las técnicas de individualización, tenemos las tutorías individuales y de grupos que se apoyan en las herramientas de comunicación, y las relacionadas con los trabajos individuales y actividades de evaluación con base en las herramientas del estudiante.

En lo relacionado con los tipos de materiales, también los estudiantes utilizan de forma muy escasa estas herramientas. A pesar de este poco uso, el método expositivo se apoya en las herramientas de comunicación para poner a disposición de los estudiantes las imágenes, dibujos y fotografías, y a través de las herramientas del curso les proporciona la guía o programas de la asignatura. La dinámica de grupos, emplea las herramientas 
del estudiante para establecer actividades en las listas o foros de discusión así como en tutoriales. Los modelos y actividades en las simulaciones se hacen posibles con las herramientas del estudiante, en donde se les coloca las prácticas y los materiales multimedia interactivos. La individualización, se apoya en las herramientas del estudiante para realizar las presentaciones de la clase y los mapas conceptuales, y con las herramientas del curso se hacen llegar los documentos y temas específicos del curso así como los esquemas de contenido.

En los procedimientos de evaluación tampoco observan un uso significativo por parte de los estudiantes. Sin embargo, dentro de este escaso uso, las herramientas de comunicación apoyan al método expositivo para valorar el aprendizaje a través de los blogs y podcasts. La dinámica de grupos emplea las herramientas del estudiante para los trabajos colaborativos así como lo hacen las simulaciones para la resolución de problemas. En el caso de la individualización, la evaluación la realiza a través de las herramientas del curso para la entrega de trabajos/actividades así como para el seguimiento de accesos a la plataforma. En tercer lugar, queremos destacar, aspectos como la dinámica del aula, las actitudes y la formación del uso de plataformas de Teleformación. Con respecto a la dinámica del aula, es relevante la percepción que tienen los estudiantes con respecto al aumento que encuentran en la dinámica de los grupos, el incremento en la comunicación entre ellos, en el trabajo autónomo y en la participación en el salón de clases, a partir del uso que han realizado de las plataformas de Teleformación. En el apartado de las actitudes, señalar, al mismo tiempo, que los estudiantes tienen una actitud que se encuentra entre un nivel de acuerdo entre la indiferencia y el estar parcialmente de acuerdo, siendo lo más destacable, que consideran que el internet es un recurso valioso para la docencia, que es bueno utilizar los recursos tecnológicos para la educación, que es conveniente formarse en el uso de las plataformas de Teleformación y que éstas pueden apoyar a cambiar el futuro de la educación superior. Por último, en cuanto a la formación en el uso de las plataformas de Teleformación, la satisfacción es baja y dentro de ésta encontramos con un mayor uso la formación en el propio centro y cursos en la universidad. Asimismo, a pesar del bajo conocimiento y de la baja importancia de las competencias formativas, las herramientas de interacción de las plataformas de Teleformación que más usan son la participación en foros/chats y las utilizadas para la entrega de trabajos/ actividades.

En resumen, los estudiantes de primer año de la Facultad de Ciencias de la Educación solo usan de forma significativa las herramientas de comunicación, en lo relacionado a otro tipos de herramientas y estrategias didácticas son poco significativo el uso que le dan, lo que indica que no acceden a la universidad con el desarrollo adecuado de habilidades tecnológicas básicas como apoyo a su proceso de formación.

\section{Conclusiones y propuestas.}

Las conclusiones de la presente investigación se estructuran en base a las hipótesis planteadas en el mismo.

Con respecto a la hipótesis 1 , en la que se planteaba que existe una relación positiva significativa entre la actitud que tiene el alumnado de primer año de la Facultad de 
Ciencias de la Educación de la UCO hacia la plataforma de Teleformación Moodle y las habilidades tecnológicas que poseen, los resultados obtenidos lo confirman, siendo que los estudiantes de primer año poseen un nivel de desarrollo significativo de las habilidades que se consideran necesarias para la Teleformación y para la formación semipresencial.

En relación a la hipótesis 2, referente a la existencia de una relación positiva significativa entre la actitud que tiene el alumnado de primer año de la Facultad de Ciencias de la Educación de la UCO y la plataforma de Teleformación Moodle con respecto a los procesos técnicos de la misma, los resultados han apuntado que los estudiantes de primer año y los docentes, observaron una actitud positiva hacia los procesos técnicos de la PTM, tanto en las herramientas que apoyan el aprendizaje del alumnado como en aquellos instrumentos que dan soporte al proceso educativo. Asimismo, se ha encontrado que la disponibilidad de recursos en la PTM quedó determinada por la visión que el docente tuvo del estudiante, así como de la estructura y organización que consideró podía establecer en su asignatura a partir del apoyo de la PTM.

La hipótesis 3, en la que se planteó la existencia de una relación positiva significativa entre la actitud que tiene el alumnado de primer año de la Facultad de Ciencias de la Educación de la UCO y la plataforma de Teleformación Moodle con respecto a los procesos didácticos que esta posee, los datos han indicado que los estudiantes percibieron que el uso de la PTM les permitió tener un proceso educativo más dinámico, sencillo y versátil, debido a que la metodología y la estructura del curso era mucho más sencilla de identificar en la PTM y facilitaba la consulta continua de materiales y participaciones en foros.

En cuanto a la retroalimentación, los estudiantes opinaron positivamente a la aclaración de dudas de la valoración de los trabajos solicitados por los docentes a través de la PTM.

La última hipótesis planteada (hipótesis 4) relativa a la existencia de una relación positiva significativa entre la actitud que tiene el alumnado de primer año de la Facultad de Ciencias de la Educación de la UCO y la plataforma de Teleformación Moodle con respecto a ser un apoyo en el proceso de enseñanza-aprendizaje, se ha encontrado en el presente estudio queel estudiante de primer año otorgó una importancia significativa y beneficiosa al uso de la PTM como elemento de apoyo al proceso educativo, perspectiva que también fue compartida por los docentes.

\section{Fuentes de financiación.}

La presente investigación ha sido subvencionada por el Gobierno de México a través de Conacyt.

\section{Referencias bibliográficas.}

Alvaréz, M. (2009). Utilizando las TIC en el aula en Blog Integración TIC en la educación, Recuperado de http://www.telefonica.net/ web2/formaciontic/metodos\%20didacticos/ estrategias_didact.html

Boneu, J.M. (2007). Plataformas abiertas de e-learning para el soporte de contenidos educativos abiertos. Revista de Universidad y Sociedad del Conocimiento, 4 (1),36-47. Recuperado de http://www.uoc.edu/rusc/4/1/ dt/esp/boneu.pdf 
Dougiamas, M. (2004). Moodle: A virtual learning environment for the rest of us. TESLEJ, 8(2), 1-8.

García, E., Gil, J. \& Rodríguez, G. (1995). Introducción a la teoría clásica de los tests. Sevilla: Grupodelta.

Gisbert, M., Cabero, J. \& Llorente, M. (2007). El papel del profesor y el estudiante en los entornos tecnológicos de formación.En J. Cabero (Coord.). Tecnología Educativa (pp.263-280). España: Mc Graw Hill.

Herrera, J.T. (2002). Delimitación conceptual de la educación abierta, a distancia y virtual. Memoria del II Congreso Internacional de educación abierta y a distancia. México: ANUIES, CICESE, UABC.

Landeta, A. (2007). Buenas Prácticas de elearning. Madrid: ANCED.

Llorente, M.C. \& Cabero, J. (2009). La formación semipresencial a través de redes telemáticas (BLended - Learning). España: Da Vinci

Marín, V. \& Maldonado, G.A. (2010). El alumnado universitario cordobés y la plataforma virtual Moodle. Pixel-Bit, Revista de Medios y Educación, 38, 121-128.

Marqués, P. (1999). Roles actuales de los estudiantes. Departamento de Pedagogía Aplicada, Facultad de Educación, UAB. Recuperado de http:// peremarques.pangea.org/estudian.htm

Marqués, P. (2011).El impacto de la sociedad de la información en el mundo educativo. Departamento de Pedagogía Aplicada, Facultad de Educación, UAB. Recuperado de http://www.dewey.uab.es/ pmarques/competen.htm

Varis, T. (2005).New Literacies and elearning competences. Recuperado de http:/ /www.elearningeuropa.info/pt/node/2328

Vázquez, A.I. (2011). Relación entre los enfoques de aprendizaje y el desempeño de los alumnos en la enseñanza presencial apoyada por plataforma educativa. Estudio de la percepción de los alumnos (Tesis doctoral inédita). Sevilla: Universidad de Sevilla.
Fecha de recepción: 20-03-2015

Fecha de evaluación: 23-03-2015

Fecha de aceptación: 16-04-2015 